\title{
Soft Tissue Infection in Intravenous Drug Users-Current Challenges
}

\author{
Faiza Rezwan $^{1 *}$, Felix Dafhnis-Calas ${ }^{2}$, Naqash Masood $^{3}$ \\ ${ }^{1}$ School of Science and Technology, Nottingham Trent University, 50 Shakespeare St, Nottingham NG1 4FQ, United Kingdom \\ ${ }^{2}$ Queens Medical Centre, University of Nottingham, Nottingham NG7 2RD, United Kingdom \\ ${ }^{3}$ School of Life Sciences, Keele University, Keele, Newcastle ST5 5BG, United Kingdom
}

DOI: $\underline{10.36348 / \mathrm{sjpm} .2020 . \mathrm{v} 05 \mathrm{i} 02.014}$

| Received: 21.01.2020 | Accepted: 28.01.2020 | Published: 29.02.2020

*Corresponding author: Faiza Rezwan

\section{Abstract}

Skin and soft tissue infections (SSTI) are the most common infections encountered in community and healthcare settings. There are different risk factors associated with SSTI including trauma, surgery, skin disease, immune defects and injection drug usage. Intravenous drug users (IDU) are frequently susceptible to SSTI due to unsafe injection practices, sharing of needles and low immunity. Intravenous drug usage is most common in young and homeless people globally. Although Staphylococcus aureus and Streptococcus pyogenes are the commonest pathogens however there are serious infections caused by rare pathogens such as botulism by Clostridium botulinum and tetanus by Clostridium Tetni. Prompt diagnosis and accurate management at the healthcare level will help in alleviating the symptoms and improve the outcomes. The continuous education and enhancing the implementation of prevention strategies are the responsibilities of local and national government to reduce the harmful drug use. In this review we are addressing the current challenges associated with causative pathogens for SSTI in IDU and their management and preventive strategies.

Keywords: SSTI Skin and soft tissue infections, IVDU-intravenous drug users.

Copyright @ 2020: This is an open-access article distributed under the terms of the Creative Commons Attribution license which permits unrestricted use, distribution, and reproduction in any medium for non-commercial use (NonCommercial, or CC-BY-NC) provided the original author and sources are credited.

\section{SSTIS AND THEIR CLINICAL PERSPECTIVE}

Skin and soft tissue infections (SSTI) are the most frequently encountered infections in healthcare settings and in community worldwide. Infections range from uncomplicated localized infections to severe and fatal infections leading to septicemia. Localized infections comprise of superficial impetigo, folliculitis, deep seated carbuncle, furuncles and abscess [1]. While some of the infections can have systemic manifestations like cellulitis and life threatening necrotizing fasciitis. Necrotizing soft tissue infections (NSTIs) are severe rapidly-spreading, soft tissue infections of the subcutaneous tissue, fascia, or muscle mostly caused by bacteria [1].

The most common pathogens that cause SSTIs are Staphylococcus aureus and Streptococcus pyogenes $[1,2]$. Both of these organisms hold various virulent factors including proteolytic enzymes, toxins and hemolysins such as beta hemolysin which support the bacteria in causing the deeper and more severe soft tissue infections [2]. In a less extend, life-threatening pathogens such as Clostridium tetani, Clostridium botulinum and Bacillus anthrax have also been reported as causal agent of SSTIs. In addition, people who inject drugs (PWID) are also susceptible to viral and bacterial infections that increase the morbidity and mortality associated with SSTIs. Among these pathogens, HIV, Hepatitis B and C viruses are the most common one [3, 4].

The major risk factors for SSTIs are the elements which lead to impairment of skin integrity as trauma, surgery, foreign body implant, intravenous catheters, skin diseases, injections and animal bites [2]. Intravenous drug users are mainly susceptible to SSTIs due to unsafe and unsterile injection practices (2). According to drug report 2017, UK has the highest number of drug users in Western Europe due to sharing of needles $[3,8]$.

Currently the major challenge for local and public health authorities is to evaluate the rare and complicated pathogens and risk of infections and its correct management. Second major challenge is to prevent the infections in drug users by reducing the drug usage especially in high risk individuals. In this review we are highlighting the current challenges associated with causative pathogens for SSTI in IDU and their management and preventive strategies. 


\section{EPIDEMIOLOGY}

Soft skin tissue infections are one of the most common reasons of hospitalization for PWID [4]. About $70 \%$ of injectors did have past story of SSTIs and only $12-35 \%$ reported current infections [5, 6]. Hospital-admission rate have doubled in the USA between 1993 and 2010. Similarly, in the UK the hospitalization due to drug injection have increased annually since 2012. This growing trend of SSTIs has caused a significant increase of the economic burden of the disease. In the United Kingdom, for example, costs to the health care system were estimated to be about $£ 77$ million per annum between July 2005 and July $2009[3,7]$.

Over the past few decades there has been a dramatic increase in number of people who inject drugs worldwide. According to Degenhardt [7] globally there are 15.6 million people who inject drugs (PWID) aged 15-64 years, with $3 \cdot 2$ million (1.6-5.1 million) women and 12.5 million (7.5-18.4 million) men. Australia has the highest percentage of women who inject drugs as $33.4 \%$ while North America and South Asia has $30 \%$ and $3.1 \%$ respectively [7]. Country wise prevalence of IV drug use is shown in Table-1.

According to epidemiologic data, demographic characteristics for vulnerable population include mostly young people less than 25 years age and homeless individuals. The other major characteristics comprise of people with history of arrest, incarceration and unsafe sexual practices. The opioids are the main drug used globally [7].

Table-1: Country wise prevalence of IDUs; intravenous drug users (adapted from Degenhardt et al., (2017) [7]

\begin{tabular}{|l|l|l|}
\hline Continents & $\begin{array}{l}\text { Number of countries with IDU } \\
\text { Estimates 2017 }\end{array}$ & Population prevalence of IDU \\
\hline Eastern Europe & 17 & $1-30 \%$ \\
\hline Western Europe & 31 & $0-34 \%$ \\
\hline East and southeast Asia & 16 & $0-25 \%$ \\
\hline South Asia & 9 & $0-9 \%$ \\
\hline Central Asia & 5 & $0-63 \%$ \\
\hline Latin America & 19 & $0-46 \%$ \\
\hline North America & 2 & $1-6 \%$ \\
\hline Sub-saharan Africa & 36 & $0-28 \%$ \\
\hline Australasia & 2 & $0-59 \%$ \\
\hline Middle east and north African & 21 & $0-12 \%$ \\
\hline Carribean & 6 & $0-44 \%$ \\
\hline Pacific island & 15 & $0-33 \%$ \\
\hline Global & 179 & $0-33 \%$ \\
\hline
\end{tabular}

The major socio demographic and risk characteristics of PWID globally comprise young people $<25$ years, recent homelessness or unstable housing, individuals with history of arrest, history of incarceration and unsafe sexual practices. In addition, it is has been estimated that globally about $83 \%$ of PWID mainly inject opioids while $33 \%$ mainly inject stimulants [7]. In the UK; opioids, cannabis and cocaine have been reported as the main injected drugs followed by amphetamines $[8,9]$."

This growing trend of SSTIs has led to a significant increase of the economic burden of the disease $[10,11]$. Recent studies has also showed that about $70 \%$ of injectors did have past story of SSTIs and only $12-35 \%$ reported current infections $[5,6,10,11]$. The recurrence, severity and duration of the SSTIs is associated with repeat hospital visits, poor antibiotic adherence, surgical intervention, hospital discharge against medical advice and social factors such as homelessness" [10,11].

The most common risk factors associated with soft tissue infections in IVDU include sharing of needles, homelessness, low immunity, presence of associated viral infections (HIV, Hep B, Hep C), unsterile injection practices, improper infection control precautions and use of acid with drugs at injection site $[8,9]$. In addition, the frequency of drug use, type of drugs, use of multiple drugs and site of injection had been cited as factors that increase the chance of developing SSTI [10]. A recent report from Tamir et al., have shown that vaso-constricting drugs such as cocaine, injected either alone or in combination with heroin may induce vasospasm and thrombosis, leading to tissue necrosis and severe infection [28]. Similarly, a new study from Public Health England indicates that injection in femoral vein has been associated with more infections as compared to arm veins $[8,9]$.

\section{Common causative pathogens for SSTIs in Intravenous Drug Users (IDU)}

The most common pathogens isolated are Staphylococcus aureus, Streptococcal pyogenes, and oral bacteria, such as Streptococcus anginosus, Fusobacterium nucleatum, Prevotella species, Porphyromonas species, and Eikenella corrodens [2]. Infection with oral pathogens is generally the 
consequence of IDU "cleaning" their needles with saliva prior to injection [12]. In addition, there are some unusual pathogens associated with SSTIs encompassing Clostridium and Bacillus species [8, 9].

There is an overall increase in the cases of group A streptococcal infections among IVDU in UK. Especially in 2017145 cases have been reported which is quite high as compared to 76 cases reported in 2016. There is also increase in cases of Methicillin resistant staphylococcus aureus MRSA and Methicillin susceptible staphylococcus aureus MSSA infections as reported in 2017. With regard to rare pathogens such as Clostridium botulinum, Clostridium tetani and Bacillus anthracis, only two cases of botulism have been reported from Scotland in 2017 while no cases of tetanus and anthrax have been documented [8-10].

\section{Staphylococcus Aureus}

Staphylococcus aureus is potentially the most virulent organism associated with variety of human infections involving different sites of body encompassing skin and soft tissue, bone, joints lungs and heart $[12,13]$. It is the most commonly isolated organism in SSTI in IVDU results from introduction of skin flora invading the epidermis and reaching the deeper tissues. Infections caused by Staphylococcus aureus can range from mild to severe localized infections or bacteremia and systemic infections leading to serious manifestations and fatal complications. There is a growing body of literature that recognizes the importance of clinical infections caused by Staphylococcus aureus because it has numerous virulence factors including proteases, toxins and hemolysins which play a significant role in causing infectious diseases [13].

Beside virulence factors, treatment options are also the major concern with Staphylococcus aureus. PWID are significantly affected by the infections caused by Methicillin resistant Staphylococcus aureus (MRSA). According to research published in the U.S. Centers for Disease Control, invasive MRSA is 16 times more likely to occur among PWID. The researchers found a positive association between the MRSA infection and non-sterile injection drug. The study also reported an increase in the prevalence of MRSA cases from $4.1 \%$ to $9.2 \%$ in the period between 2011 and 2016 [14]. Methicillin resistant Staphylococcus aureus (MRSA) infections are the key issue globally. In UK there is mandatory monitoring of cases with MSSA (Methicillin susceptible staphylococcus aureus) bacteremia and MRSA bacteremia and one report shows prevalence of $11 \%$ in intravenous drug users [8].

\section{Streptococcus Pyogenes}

Group A Streptococcus (GAS) causes a wide variety of diseases extending in severity from uncomplicated pharyngitis to fatal necrotizing fasciitis and streptococcal toxic shock syndrome [15]. Currently there is an upsurge in infections because of the introduction of unusual and novel strains in IVDU and due to the advancement in whole genome sequencing, typical strains can now be identified and discovered [16]. The outbreak of Streptococcal pyogenes cases have been reported recently in southwest England [15]. The increased incidence of iGAS was also been seen on a very specific population of IVDU described by Taryn \& Athey et al., in their study [16].

\section{Clostridium Botulinum}

Wound botulism is a rare but very serious illness caused by a neurotoxin secreted by Clostridium botulinum. It is a spore forming bacteria and botulinum spores are found naturally in the environment (dust, soil) so they can potentially contaminate supplies of drugs such as heroin. In UK, the sporadic cases of wound botulinum among IVD have been reported over the last decade due to the contamination of heroin with botulinum spores from environment and are thought to be a continuing problem [17]. Wound botulism could develop by injecting contaminated drugs through the skin. The botulinum toxin is absorbed in the blood and leads to various clinical symptoms including double vision, difficulty in speaking and swallowing, extreme weakness and paralysis that can affect the arms, legs and lungs [17]. Most cases make a full recovery if treated appropriately; otherwise mortality rate is $5-10 \%$ worldwide [18]. Botulism is treated by giving botulinum antitoxin, which can reduce the severity of the symptoms if administered early in the course of the disease and appropriate antibiotics with proper wound cleaning $[17,18]$.

\section{Clostridium Tetani}

Tetanus is caused by potent neurotoxin produced by clostridium tetani which enters the body through a wound or through injecting drugs. Its spores are present in soil or in contaminated drugs. The incubation period is around 10 days but it can be prolonged to 3 weeks or months. Infection may be due to the injecting equipment being contaminated with spores from the soil or from contaminated drugs [19]. Symptoms include lock jaw and spastic paralysis of upper and lower extremities. In severe cases they may cause respiratory difficulties. It is a fatal infection and needs prompt diagnosis and treatment with tetanus immunoglobulin and antibiotics with proper wound cleaning. It can be prevented by tetanus toxoid vaccination [20].

\section{Clostridium Novyi, Clostridium Sordellii, Clostridium Perfringens}

They are Gram positive spore forming anaerobic rods and can lead to soft tissue infections by secreting toxins. Although ubiquitous, C. novyi is only rarely reported as a pathogen, contributing to mixed infections in contaminated traumatic wounds. The injecting drug users used heroin subcutaneously with 
citric acid, and it is likely that the presence of foreign material facilitated the establishment of Clostridium infection in these patients [21]. Black tar heroin is often mixed with dirt that may be contaminated with heatresistant spores. It is often injected into the subcutaneous tissue or dermis, an anaerobic environment where the spores are able to germinate and release exotoxin into the surrounding tissues and cause gas gangrene [22].

\section{Bacillus Anthracis}

It is a Gram positive spore forming aerobic rod which is associated with cutaneous gastrointestinal and respiratory anthrax on the basis of route of entry. Spores can be found in animal products such as wool, hair, hides, skins, bones and in the carcasses of infected animals. The spores can also contaminate soil and may survive for many years [23]. In cutaneous anthrax, a lesion appears on the skin and develops into a characteristic ulcer with a black centre. Inhalational anthrax begins with a flu-like illness and is followed by respiratory compromise and shock around two to six days later. Intestinal anthrax results in severe abdominal pain, fever and bloody diarrhoea [23]. Injectional anthrax has a variety of clinical presentations which is different from three usual clinical presentations. Mostly cases do not present with the characteristic features of cutaneous anthrax with classical black eschar, but in most cases with marked soft tissue oedema and often with severe pain [24]. There are two cases reported by Russell et al., in which one case had been diagnosed very late due to unusual and severe systemic presentation [25].

\section{Clinical Spectrum}

The clinical manifestations for soft tissue infections in IVDU are as inexplicable and diverse as causative pathogens. Abscesses and cellulitis include the most common SSTI affecting IDU. Abscess is the collection of pus while cellulitis is the infection of dermis and subcutis that leads to rapidly spreading areas of erythema, swelling, tenderness, and warmth, sometimes accompanied by lymphangitis and inflammation of the regional lymph nodes $[1,2]$.

Systemic symptoms can occur in moderate to severe cases of cellulitis including fever, leukocytosis and hypotension [2]. There are several independent risk factors linked to SSTI in injection drug abusers, as discussed earlier. In addition to unsafe injection practices skin popping is also considered to be the main risk factor associated with abscesses and cellulitis [2]. Methamphetamine alters the antimicrobial efficacy of phagocytic cells during MRSA induced skin infection, reduces the wound healing and also induces biofilm formation illustrated in study done by Mihu et al., [26]. Abscesses and chronic wounds are significant causes of morbidity for injection drug users (IDUs) and also the primary reason for hospitalization [2]. Variable sites of abscess are also commonly seen in IDU, including neck, groin, tongue, arms legs depending upon the route of injection [27]. Bundle et al., reported an outbreak of invasive and non-invasive disease due to an unusual type of Streptococcus pyogenes, emm66 among drug users, largely homeless population in Southern England and Wales, identified in September 2016. A total 27 cases had been reported of which 20 indicated soft tissue infections including abscesses, cellulitis, and injection site infection and necrotizing fasciitis [15].

Vein injury is very common among IVDU due to non-sterile injection and direct toxic effects of the drug and can lead to chronic venous insufficiency, which increases the risk for chronic lower extremity ulcers [28]. Wounds with chronic venous insufficiency can also lead to more severe SSTIs, including necrotizing fasciitis, gangrene and sepsis, potentially resulting in amputation or death [29]. Necrotizing fasciitis is a fatal and destructive subcutaneous infection which goes along the superficial fascia and can lead to complication in deeper tissues and gangrene. Illicit drug use can also be linked to another common skin and softtissue manifestation, vasculitis and it has been reported with cocaine-induced midline destructive lesions, such as nasal septal and palatal perforations, due to snorting cocaine [28, 29]. Recurrent infections are also the major issue with IVDU due to poor adherence to antibiotics and hospital discharge against medical advice and homelessness [30, 31].

\section{Diagnosis}

The diagnosis of most SSTIs is based on clinical criteria although laboratory investigations and imaging studies help to confirm the diagnosis and elucidate characteristics of specific etiologies. The clinical criteria are not easily evaluated and most of the time is a real challenge to differentiate between the necrotizing and the not necrotizing infections. Hence careful assessment of the wound is needed to discriminate among superficial and deep necrotizing infections.

Even though microbiological information do not share a part in the selection of initial empiric therapy, the requirement for aetiological tests depends on some factors, including severity of the clinical condition; type of infection; and the underlying patient condition. Cultures are not necessary for common and simple SSTIs (cellulitis or small subcutaneous abscess), on the contrary when complicated SSTIs are associated with exudates or with abscesses, specimens have to be sent promptly to microbiology laboratory with detailed history. Pus is aspirated for microscopic examination and culture in cases of abscess [32]. The blood culture positivity is around $5 \%$ and needle aspirate culture positivity is around $5-40 \%$ in cellulitis therefore aspirate or tissue sample is only taken in severe cases or if there is open wound [33].PCR techniques does not seem to be ideal but it can be considered in cases of MRSA infections. 
Necrotizing fasciitis have systemic manifestations therefore blood culture and complete blood count with CRP (C reactive protein and ESR (Erythrocyte sedimentation rate) is considered. Furthermore deep tissue sample is taken for bacteriological culture [33].

Imaging techniques also play a major role in diagnosing SSTI. Radiography will able to detect gas in soft tissues and bone involvement suggesting necrotizing infection and osteomyelitis. MRI (Magnetic resonance imaging) is very useful in differentiating cellulitis from abscess and in detecting necrosis, edema and deep fascial involvement [34]. However due to the high cost of MRI; nowadays ultrasound is playing a major role in supporting the diagnosis of SSTI. Ultrasound is not only easy and rapid to perform but it is also beneficial for therapeutic aspiration in cases of abscess preventing complications and unnecessary interventions [33, 34].

\section{Treatment}

Management of SSTI in IVDU needs proper evaluation of the site of infection and assessment of the severity. Relevant questions regarding type, preparation and frequency of drugs would also be important in case management. According to IDSA abscess needs incision and drainage in mild cases but in moderate to severe cases with fever antibiotic therapy is suggested [35]. Flucloxacillin and cefazolin are the main antibiotics used worldwide as first line deference for five days. Erythromycin, amoxicillin-clavulunate, clindamycin are some of the alternative antibiotics recommended in case of abscesses [35]. When suspicion of MRSA is high then vancomycin or linezolid is suggested [35]. Daptomycin and ceftaroline are also mentioned in soft tissue infections by MRSA.

Dalbavancin, oritavancin and tedizolid are new drugs, approved or in development for SSTI treatment, that also proved to be efficient against MRSA. Dalbavancin and oritavancin have a long half-life and can be dosed less frequently, hence can be given on outpatient basis [29].

In mild cases of cellulitis, penicillin as oral therapy can be given while in moderate to severe cases intravenous initial aminopenicillin plus a betalactamase inhibitor or moxifloxacin, possibly plus clindamycin are prescribed [1]. Outpatient treatment is the main issue for IVDU as they are not compliant with medications therefore sometimes hospitalization is needed in these cases $[32,36]$.

$$
\begin{aligned}
& \text { Box 1: Key Facts } \\
& \text { - One third of PWD report having a recent } \\
& \text { symptom of bacterial infections and related } \\
& \text { outbreaks continue to occur in this vulnerable } \\
& \text { group. } \\
& \text { - Abscess, cellulitis and ulcers are the most common } \\
& \text { SSTI among PWD. } \\
& \text { - The level of needle sharing has come down in UK } \\
& \text { but still it is a problem and almost } 1 \text { in } 6 \text { persons } \\
& \text { reported to share needles. } \\
& \text { Prevention is aimed at reducing the illicit drug use, } \\
& \text { provision of effective interventions to reduce the } \\
& \text { risk of infections and proper recovery measures for } \\
& \text { affected individuals. }
\end{aligned}
$$

Necrotizing fasciitis needs aggressive and urgent management with surgical exploration, intravenous broad-spectrum antibiotic with symptomatic treatment including analgesics and antiinflammatory drugs. Vancomycin with piperacillin, tazobactum, carbapenem or ceftriaxone in combination with metronidazole is recommended by IDSA as it covers the Gram positive, Gram negative organisms and anaerobes. Penicillin with clindamycin is given in case of streptococcal necrotizing fasciitis as clindamycin also has inhibitory effect on its toxin. In a similar way gangrene also needs urgent treatment with broadspectrum antibiotics covering Gram positive and Gram negative organisms. Penicillin with clindamycin can also be given in case of clostridial myonecrosis [35]. 


\section{Prevention Strategies}

Prevention and reduction in drug use among vulnerable individuals and building up of healthier society is the responsibility of any government at both national and local levels with community sector. The impact of serious and often fatal infectious diseases among IVDU can be reduced by strengthening the policies that provide access to sterile injection equipment, reform the education regarding injecting drug usage and management for substance use disorder [37]. The UK have Drug strategy plan to reduce the drug use and adverse effects related to it. Its overall aims are to decrease all illicit and other harmful drug use by adopting the smarter approach to restrict the supply of drugs: and increase the rate of individuals recovering from their dependence by improving both treatment quality and outcomes. Other areas which have been covered by strategy plan are the provision of healthcare, education, housing and social care $[36,37]$.

Furthermore for the prevention of infection there are awareness programs about safe injection practices and reduction of sharing of needles conducted by health authorities globally [37]. The other interventions which have been practiced worldwide include provision of free needles, syringes and other equipment; promotion of safe disposal of used equipment; infection counseling; support and testing; vaccinations against $\mathrm{HBV}$ and tetanus; referral to drug treatment; treatment for HIV and HCV infection; and the provision of take-home naloxone and training of drug users and their family members on its use [37]. There are also needle syringe programs which educate people about infection control, infection to high risk area, proper disposal of needles and syringes and use of low dead space equipment [38].

With the growing concern of recent increase in HIV cases among IDUs in Pakistan, countrywide dropin centers were established in major cities of Pakistan giving them education about needle exchange, adverse health effects of drug use and counseling for HIV. The major barrier in prevention for resource limited countries like Pakistan is the non-availability of opioid substitution treatment and Methadone therapy [39]. Samo et al., highlighted other hurdles in conducting risk reduction program including loss of follow-up as high percentage of IDUs are uneducated, young and homeless individuals [40]. UK Department for International Development and United Nations Office of Drugs and Crime has also supported the establishment of harm reduction services in major cities of Pakistan [39, 40].

Various studies worldwide recommended using soap and water or alcohol pads prior to injecting drugs [36, 37]. According to Harris et al., the major barriers in practicing risk reduction included lack of supplies and withdrawal symptoms [11]. Moreover there are wound care services and urgent referral in UK which can help in the proper management of cases of soft tissue infections and prevention of fatal complications [38].

\section{Outstanding questions}

- How can we prevent soft tissue infections in intravenous drug users and is the current strategy plan enough?

- Should we implement more surveillance programs to monitor soft tissue infections?

- Can more research be done to evaluate the barriers in risk reduction of SSTI?

\section{Concluding Remarks}

Globally there is alarming increase in the number of injection drug users resulting in upsurge of soft tissue infections. The blood borne virus infections along with soft tissue infections are triggering the increase in morbidity and mortality in IVDU. Prompt diagnosis and accurate management will help in alleviating the symptoms and improve the outcomes.

The continuous education and enhancing the implementation of prevention strategies at local and national level will also cut down the harmful drug use. Proper recovery and rehabilitation of affected individuals and strong application of drug act to limit the availability of drugs would be beneficial to the society in future.

Countries like UK have data monitoring systems but it can be done more frequently especially for the soft tissue infections in IVDU. Future research and studies are warranted to evaluate the barriers in practicing risk reduction behavior and in accessing SSTI care among IVDU.

\section{REFERENCES}

1. Sunderkötter, C., \& Becker, K. (2015). Frequent bacterial skin and soft tissue infections: diagnostic signs and treatment. JDDG: Journal der Deutschen Dermatologischen Gesellschaft, 13(6), 501-526.

2. Smith, M. E. (2015). High Rates of Abscesses and Chronic Wounds in Community-Recruited Injection Drug Users and Associated Risk Factors. Journal Addict Med. 9(2):87-93.

3. Emcdda.europa.eu. (2019). [online] Available at: http://www.emcdda.europa.eu/system/files/publica 
tions/4529/TD0116925ENN.pdf_en [Accessed 05 Apr. 2019].

4. Dahlman, D., Jalalvand, F., Blomé, M., Håkansson, A., Janson, H., Quick, S., \& Nilsson, A. (2016). High Perineal and Overall Frequency of Staphylococcus aureus in People Who Inject Drugs, Compared to Non-Injectors. Current Microbiology, 74(2), 159-167.

5. Dahlman, D., Håkansson, A., Kral, A., Wenger, L., Ball, E., \& Novak, S. (2016). Behavioral characteristics and injection practices associated with skin and soft tissue infections among people who inject drugs: A community-based observational study. Substance Abuse, 38(1), 105112.

6. Smith, T. (2015). Livestock-Associated Staphylococcus aureus: The United States Experience. PLOS Pathogens, 11(2), e1004564.

7. Degenhardt, L., Peacock, A., Colledge, S., Leung, J., Grebely, J., Vickerman, P., ... \& Lynskey, M. (2017). Global prevalence of injecting drug use and sociodemographic characteristics and prevalence of $\mathrm{HIV}, \mathrm{HBV}$, and $\mathrm{HCV}$ in people who inject drugs: a multistage systematic review. The Lancet Global Health, 5(12), e1192-e1207.

8. PHE Shooting Up: Infections among people who inject drugs in the UK, 2017 An update, November 2018. https://www.gov.uk/government/publications/shoo ting-up-infections-among-people-who-injectdrugs-in-the-uk [Accessed 12 Apr. 2019]

9. Public Health England (PHE). Unlinked Anonymous Monitoring Survey of People Who Inject Drugs in contact with specialist services: data tables. Surveillance Update: July 2016. London: PHE. Available from: https://www.gov.uk/government/ [Accessed 10 Apr. 2019]

10. HM Government. 2017 Drug Strategy London July 2017 [Available from: https:/www.gov.uk/government/uploads/system/u ploads/attachment_data/file/628148/Drug_strategy _2017.PDF. [Accessed 8 Apr. 2019]

11. Harris, M., Brathwaite, R., McGowan, C. R., Ciccarone, D., Gilchrist, G., McCusker, M., ... \& Hope, V. (2018). 'Care and Prevent': rationale for investigating skin and soft tissue infections and AA amyloidosis among people who inject drugs in London. Harm reduction journal, 15(1), 23.

12. Raiker, N., Aouthmany, M., \& Ezra, N. (2016). Dermatologic signs and symptoms of substance abuse. J Clin Exp Dermatol Res, 7, 337.

13. Tong, S. Y., Davis, J. S., Eichenberger, E., Holland, T. L., \& Fowler, V. G. (2015). Staphylococcus aureus infections: epidemiology, pathophysiology, clinical manifestations, and management. Clinical microbiology reviews, 28(3), 603-661.

14. Jackson, K., Bohm, M., Brooks, J., Asher, A., Nadle, J., Bamberg, W., Petit, S., Ray, S.,
Harrison, L., Lynfield, R., Dumyati, G., Schaffner, W., Townes, J., \& See, I. (2018). Invasive Methicillin-Resistant Staphylococcus aureus Infections Among Persons Who Inject Drugs Six Sites, 2005-2016. MMWR. Morbidity and Mortality Weekly Report, 67(22), 625-628.

15. Bundle, N., Bubba, L., Coelho, J., Kwiatkowska, R., Cloke, R., King, S., ... \& Lamagni, T. (2017). Ongoing outbreak of invasive and non-invasive disease due to group A Streptococcus (GAS) type emm66 among homeless and people who inject drugs in England and Wales, January to December 2016. Eurosurveillance, 22(3).

16. Athey, T. B., Teatero, S., Sieswerda, L. E., Gubbay, J. B., Marchand-Austin, A., Li, A., ... \& Fittipaldi, N. (2016). High incidence of invasive group A Streptococcus disease caused by strains of uncommon emm types in Thunder Bay, Ontario, Canada. Journal of clinical microbiology, 54(1), 83-92.

17. PHE Botulism: Infection in people who inject drugs. July 2012 Available at https://www.gov.uk/government/publications/botu lism-infection-in-people-who-inject-drugs [Accessed 05 Apr. 2019].

18. WHO https://www.who.int/news-room/factsheets/detail/botulism

19. Collins, S., Amirthalingam, G., Beeching, N. J., Chand, M. A., Godbole, G., Ramsay, M. E., ... \& White, J. M. (2016). Current epidemiology of tetanus in England, 2001-2014. Epidemiology \& Infection, 144(16), 3343-3353.

20. WHO. (2018). The Immunological Basis for immunization series Module 3: Tetanus Update.

21. Noone, M., Tabaqchali, M., \& Spillane, J. B. (2002). Clostridium novyi causing necrotising fasciitis in an injecting drug user. Journal of clinical pathology, 55(2), 141-142.

22. Mars, S. G., Bourgois, P., Karandinos, G., Montero, F., \& Ciccarone, D. (2016). The textures of heroin: User perspectives on "black tar" and powder heroin in two US cities. Journal of psychoactive drugs, 48(4), 270-278.

23. National Anthrax Outbreak Control Team. (2011). An Outbreak of Anthrax among Drug Users in Scotland, December 2009 to December 2010. In Health Protection Scotland.

24. Sykes, A., Brooks, T., Dusmet, M, Nicholson, A. G., Hansell, D. M., \& Wilson, R. (2013). Scottish Drugs Forum (2013). Anthrax and Heroin Users: What Workers Need to Know.

25. Russell, L. (2013). Case Report. Two anthrax cases with soft tissue infection, severe oedema and sepsis in Danish heroin users. BMC Infectious Diseases, 13:408.

26. Phillips, K. T., Altman, J. K., Corsi, K. F., \& Stein, M. D. (2013). Development of a risk reduction intervention to reduce bacterial and viral infections for injection drug users. Substance use \& misuse, 48(1-2), 54-64. 
27. Mihu, M. R., Roman-Sosa, J., Varshney, A. K., Eugenin, E. A., Shah, B. P., Lee, H. H., ... \& Martinez, L. R. (2015). Methamphetamine alters the antimicrobial efficacy of phagocytic cells during methicillin-resistant Staphylococcus aureus skin infection. MBio, 6(6), e01622-15.

28. Tamir, S. O., Marom, T., Len, A., Gluck, O., Goldfarb, A., \& Roth, Y. (2015). Deep neck infections in cervical injection drug users. The Laryngoscope, 125(6), 1336-1339.

29. Coull, A. F., Atherton, I., Taylor, A., \& Watterson, A. E. (2014). Prevalence of skin problems and leg ulceration in a sample of young injecting drug users. Harm reduction journal, 11(1), 22.

30. Wurcel, A. G., Merchant, E. A., Clark, R. P., \& Stone, D. R. (2015). Emerging and underrecognized complications of illicit drug use. Clinical Infectious Diseases, 61(12), 18401849.

31. Ciccarone, D., \& Harris, M. (2015). Fire in the vein: heroin acidity and its proximal effect on users' health. International Journal of Drug Policy, 26(11), 1103-1110.

32. UK Standards for Microbiology Investigations Investigation of Skin and Superficial Soft Tissue PHE 2016.

33. Stevens, D. L., Bisno, A. L., Chambers, H. F., Dellinger, E. P., Goldstein, E. J., Gorbach, S. L., ... \& Wade, J. C. (2014). Practice guidelines for the diagnosis and management of skin and soft tissue infections: 2014 update by the Infectious Diseases Society of America. Clinical infectious diseases, 59(2), e10-e52.

34. Esposito, S., Bassetti, M., Concia, E., De Simone, G., De Rosa, F., Grossi, P., Novelli, A., Menichetti, F., Petrosillo, N., Tinelli, M., Tumbarello, M., Sanguinetti, M., Viale, P.,
Venditti, M., \& Viscoli, C. (2017). Diagnosis and management of skin and soft-tissue infections (SSTI). A literature review and consensus statement: an update. Journal of Chemotherapy, 29(4), 197-214.

35. Russo, A., Concia, E., Cristini, F., De Rosa, F. G., Esposito, S., Menichetti, F., ... \& Viscoli, C. (2016). Current and future trends in antibiotic therapy of acute bacterial skin and skin-structure infections. Clinical Microbiology and Infection, 22, S27-S36.

36. Hutin, Y., Hauri, A., Chiarello, L., Catlin, M., Stilwell, B., Ghebrehiwet, T., \& Garner, J. (2003). Best infection control practices for intradermal, subcutaneous, and intramuscular needle injections. Bulletin of the World Health Organization, 81, 491-500.

37. Werb, D., Bluthenthal, R., Kolla, G., Strike, C., Kral, A., Uusküla, A., \& Des Jarlais, D. (2017). Preventing Injection Drug use Initiation: State of the Evidence and Opportunities for the Future. Journal of Urban Health, 95(1), 91-98.

38. Public health guideline Needle and syringe programmes. 26 March 2014

39. UNODC, UNAIDS Pakistan country advocacy brief on injecting drug use and HIV. http://www.unodc.org/documents/southeastasiaan dpacific/topics/hivaids/UNRTF/PAK_CAB_29_Mar_10_.pdf (Accessed on 2nd December 2013)

40. Samo, R., Agha, A., Shah, S., Altaf, A., Memon, A., Blevins, M., Qian, H., \& Vermund, S. (2016). Risk Factors for Loss to Follow-Up among People Who Inject Drugs in a Risk Reduction Program at Karachi, Pakistan. A Case-Cohort Study. PLOS ONE, 11(2):e0147912. 